\title{
Model reduction of homogeneous-in-the-state bilinear systems with input constraints
}

\author{
I.J. Couchman, E.C. Kerrigan and C. Böhm
}

\begin{abstract}
Homogeneous-in-the-state bilinear systems, appended by an additive disturbance, appear both from the discretization of some partial differential equations and from the bilinearization of certain nonlinear systems. They often have large state vectors that can be cumbersome for simulation and control system design. Our aim is to define a method, invariant to time transformations, for finding a reduced-order model with similar disturbance-output characteristics to those of the plant for all admissible input sequences. The inputs considered satisfy simple upper and lower bound constraints, representing saturating actuators. The approximation is based on a model truncation approach and a condition for the existence of such an approximation is given in terms of the feasibility of a set of linear matrix inequalities. A novelty of our work is in the definition of a new Gramian for this class of system. Explicit error bounds on the scheme are included. The paper concludes with a demonstration of the proposed approach to the model reduction of a solar collector plant.
\end{abstract}

\section{INTRODUCTION}

Bilinear systems are a special class of nonlinear systems that are linear in the input and linear in the state, but not jointly linear in both. They have received considerable attention because they offer something of a halfway house between linear and nonlinear models. A review of applications and properties can be found in [1], [2]. We consider a class of such systems known as continuous-time, homogeneous-inthe-state bilinear systems [1] in the presence of an additive disturbance that are multi-input multi-output (MIMO) and can be written in the form

$$
\begin{aligned}
\dot{x}(t) & =A x(t)+\sum_{i=1}^{m} N_{i} x(t) u_{i}(t)+R w(t), \\
y(t) & =C x(t), \quad x(0)=x_{0},
\end{aligned}
$$

where the state $x:[0, \infty) \rightarrow \mathbb{R}^{n}$, output $y:[0, \infty) \rightarrow \mathbb{R}^{p}$, disturbance $w:[0, \infty) \rightarrow \mathbb{R}^{r}, A, N_{i} \in \mathbb{R}^{n \times n}$ for all $i \in$ $\{1, \ldots, m\}, C \in \mathbb{R}^{p \times n}, R \in \mathbb{R}^{n \times r}$ and control input $u_{i} \in \mathcal{U}$ for all $i \in\{1, \ldots, m\}$. We consider the case where $\mathcal{U}:=\{u$ : $\left.[0, \infty) \rightarrow \mathbb{R}\left|\sup _{t}\right| u(t) \mid \leq 1\right\}$. This choice of $\mathcal{U}$ corresponds to systems where the inputs are independently constrained as a result of saturating actuators, for example. Note that simple upper and lower bound constraints can be rewritten in this form by a simple change of variable (see the example in

I.J.Couchman and E.C.Kerrigan are with the Department of Electrical and Electronic Engineering and the Department of Aeronautics, Imperial College London, Exhibition Road, London, SW7 2AZ. ian.couchman06@imperial.ac.uk, e.kerrigandimperial.ac.uk. C. Böhm is with Institute for Systems Theory and Automatic Control, University of Stuttgart, Pfaffenwaldring 9, 70550, Stuttgart, Germany. cboehmeist. uni-stuttgart.de
Section IV). Interest in such a model stems from the need for control of large-scale, input-constrained nonlinear systems of the form

$$
\begin{aligned}
\dot{x}(t) & =f(x(t))+\sum_{i=1}^{m} g_{i}(x(t)) u_{i}(t)+h(x(t)) w(t),(2 \mathrm{a}) \\
y(t) & =C x(t), \quad x(0)=x_{0},
\end{aligned}
$$

where $f, g_{i}: \mathbb{R}^{n} \rightarrow \mathbb{R}^{n}, h: \mathbb{R}^{n} \rightarrow \mathbb{R}^{n \times r}, u_{i} \in \mathcal{U}$ for all $i \in\{1, \ldots, m\}$ and $w:[0, \infty) \rightarrow \mathbb{R}^{r}$. With a linearization near an equilibrium point or the Carleman bilinearization, nonlinear systems of the form in (2) can sometimes be approximated by a system of the form in (1) [3]. There is an additional requirement on $g_{i}$ such that its linearization leads to a term strictly linear in $x$ as opposed to an affine one. Examples of such systems can be found in a range of fields including ecological systems [2], fluid mixing applications [4] and solar energy plants [5]. They may at first glance seem a peculiar class of system as they have a special property: once at the origin, the input cannot affect the system in the absence of a disturbance. Consider a white liquid with a disturbance representing the addition of a miscible red dye at a specific part of the domain. An input can stir the dye and the liquid to form a pink colour, but once the resultant liquid is pink, no amount of stirring can separate the red and white components.

Control system design for constrained, nonlinear systems is notoriously difficult. Receding horizon approaches offer one option, although they require the recursive, online solution of open-loop optimal control problems [5]. For even moderately sized systems, this can be prohibitively expensive. Generating reduced-order models for use in simulation or control of such large-scale systems has therefore been the focus of considerable research. Some work has already been carried out on model reduction of general nonlinear systems [6] and bilinear systems [7]-[9] and so are applicable to the homogeneous-in-the-state bilinear case. In [6], observability and controllability functions are defined for a general nonlinear system. To compute such quantities, the solution to a Hamilton-Jacobi-Bellman type partial differential equation must be found - something notoriously difficult to do even for small systems. Similarly, there is no reason the reduced order model would be bilinear [10]. The application of the approach from [6] to singularly perturbed bilinear systems is considered in [11]. The authors use the structure provided by a model containing simultaneously slow and fast dynamics to simplify the computation of the bilinear system Gramians although this is more a study into 
the structure of bilinear system functions than a description of a model reduction scheme. In [7]-[9] the observability and controllability Gramians as defined in [12] form the basis of the reduction scheme, but differ in the fact that [7] and [9] consider balanced truncation type approaches for model reduction, while [8] considers a frequency domain $\mathcal{H}_{2}$ based reduction method. The Gramians in [12] are defined in terms of the kernels of the Volterra series expansion of the state. A benefit of such an approach is that they can be computed as the solution of Generalized Lyapunov equations [13] of the form

$$
A^{\prime} Q+Q A+\sum_{i=1}^{m} N_{i}^{\prime} Q N_{i}+C^{\prime} C=0,
$$

which for certain types of system can be computed efficiently [13]. An interesting discussion of the physical interpretation of such a Gramian can also be found in [10]. In [7] the reduction is carried out by approximating the bilinear observability and controllability Gramians with lower-order ones via principal component analysis [14]. The balanced truncation method can be shown to perform well on numerical examples [7]. However, there are certain drawbacks not highlighted. These are summarized in Section III-A and helps motivate the new results in this paper. In order to address the problems described, we consider two new Gramians, coined the D-Gramian and E-Gramian that have simple energybased interpretations. We show that suitable examples of such Gramians can be computed as the solution of an LMI constrained optimization problem.

The main contribution of this paper is the definition of a new method for the model reduction of an homogeneousin-the-state bilinear system. This involves the discussion of some problems with an existing approach, the definition of two new Gramians, and the introduction to a reduction scheme. The new reduction scheme is invariant to model time transformations and so, unlike the existing approaches, shows no degradation in performance when the units of time used in the plant modeling are changed. This issue is discussed in detail and demonstrated on a study of a model of a solar collector plant with the new scheme outperforming an existing method for reduced order models of the same size. As a final contribution, a reduced order model similar to the solar collector plant is shown to exist, thereby motivating future work into efficient control system design for such a plant.

This paper is organized as follows. The problem is formulated in Section II. Sections III-A and III-B discuss two different definitions of Gramians about which a model reduction scheme can be based. The first is the one used in [7], the second a new construction and the paper's first contribution. In Section III-C, a new reduction scheme is proposed and important properties discussed. Section IV demonstrates the algorithm on a real-world example. Finally, some conclusions are given and future work proposed.

\section{Formal Problem Statement}

This work is focused on computing a reduced-order model with similar disturbance-output properties to those of the plant. To achieve similar disturbance-output behavior we consider minimizing the maximum $\mathcal{L}_{2}$-gain of the disturbance-error system for all feasible input sequences. A realization $\left(A, N_{1}, \ldots, N_{m}, C, R\right)$ refers to the system (1). The problem is formally written:

Sub-optimal disturbed model truncation: Given a $\gamma>0$, find a projection matrix $T \in \mathbb{R}^{q \times n}, q<n$ satisfying:

$\max _{u \in \mathcal{U}} \max _{\substack{w \in \mathcal{L}_{2}[0, \infty) \\ w \neq 0}} \frac{\int_{0}^{\infty}(y(t)-\hat{y}(t))^{\prime}(y(t)-\hat{y}(t)) \mathrm{d} t}{\int_{0}^{\infty} w^{\prime}(t) w(t) \mathrm{d} t} \leq \gamma$,

with $x_{0}=0$, where $y$ is the output of the realization $\left(A, N_{1}, \ldots, N_{m}, C, R\right), \hat{y}$ is the output of the realization $\left(T A T^{+}, T N_{1} T^{+}, \ldots, T N_{m} T^{+}, C T^{+}, T R\right)$ and $u, w$ are the inputs and disturbances, respectively, applied to both systems. $\mathcal{L}_{2}[0, \infty)$ is the spaces of square integrable and Lebesgue measurable functions defined on the interval $[0, \infty)$, and $T^{+}$represents the Moore-Penrose pseudoinverse of $T$ [15].

Note that the matrix $T$ projects the dynamics onto a subspace of $\mathbb{R}^{n}$, and so if $\hat{x}$ is the state of the reduced realization, then $T^{+} \hat{x}(t)$ is an approximation to $x(t)$. It should also be noted at this point that solutions to the suboptimal disturbed model truncation problem are generally not unique, because realizations are typically invariant to a state coordinate transformation [16]. Also note that this is a sub-optimal model reduction scheme because we are constraining ourselves to the problem of finding a truncation matrix capable of achieving the desired performance.

Section III will show that the model reduction procedure to solve the sub-optimal disturbed model truncation problem is:

1) Find a D-Gramian $P$ and an E-Gramian $Q$ using Algorithm 2 from Section III-D;

2) Find the balancing transformation $U$ and transformed Gramians $\tilde{P}=\tilde{Q}=\Sigma$ using Algorithm 1 from Section III-C;

3) Examine the singular values (diagonal components of $\Sigma$ ) and decide upon a $q$ to achieve satisfactory performance (using the error bounds of Theorem 1), then compute $T:=\left[\begin{array}{ll}I_{q \times q} & 0_{q \times(n-q)}\end{array}\right] U$;

4) Compute the reduced disturbed realization $\left(T A T^{+}, T N_{1} T^{+}, \cdots, T N_{m} T^{+}, C T^{+}, T R\right)$.

\section{SOLUTION}

The solution approach involves using results from principal component analysis (PCA) [17] to approximate a Gramian by a reduced-order counterpart. For a detailed explanation of these approaches for model reduction, see [14]. Sections III-A and III-B discuss two different definitions of the Gramian for reduction, the former being the result of balancing the well-known bilinear observability and controllability Gramians from [12] presented in [7], the second a new construction and the paper's first contribution. In the 


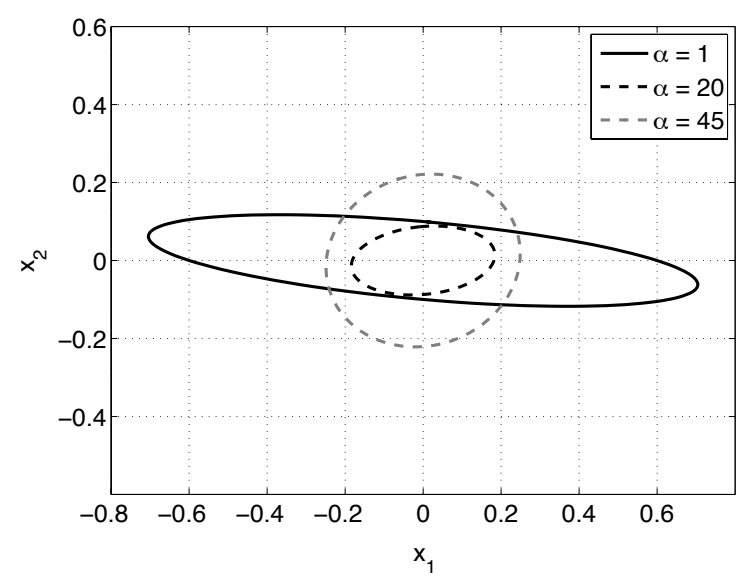

Fig. 1. Ellipses representing the bilinear observability Gramians for a range of $\alpha$ values, computed as solutions to (3)

sections that follow, " $\succ(\succeq)$ 0" denotes positive (semi-) definite and “ $\prec(\preceq) 0$ ” denotes negative (semi-) definite.

\section{A. Bilinear System Gramians}

The bilinear observability and controllability Gramians were first introduced in [12] to analyze the structural properties of bilinear systems. They are defined in terms of the kernels of the Volterra series expansion of the system state. In [13] they are shown to be easily computed as the solution of simple linear matrix equalities; for example, the bilinear observability Gramian must satisfy (3), a Generalized Lyapunov equation. The work presented in [7] then uses the bilinear observability and controllability Gramians to reduce the order of the bilinear system. In this section we show a previously unreported drawback of this approach.

Consider the time transformation $\tau=\frac{1}{\alpha} t$, hence

$$
\begin{aligned}
\dot{x}(\tau) & =\alpha A x(\tau)+\alpha \sum_{i=1}^{m} N_{i} x(\tau) u_{i}(\tau)+\alpha R w(\tau), \\
y(\tau) & =C x(\tau) .
\end{aligned}
$$

Therefore $Q$ would be the solution of

$$
\alpha A^{\prime} Q+\alpha Q A+\alpha^{2} \sum_{i=1}^{m} N_{i}^{\prime} Q N_{i}+C^{\prime} C=0 .
$$

It is not desirable for a model reduction scheme to change with the unit of time selected, because there is no reason why the most observable modes, or the ratio of how observable specific modes are, should change. However, by considering the following numerical example, this is exactly what we see for the bilinear system Gramians:

$$
\begin{aligned}
A & =\left[\begin{array}{cc}
-1 & -0.2 \\
0.2 & -1
\end{array}\right], N_{1}=\left[\begin{array}{cc}
0.1 & 0.1 \\
0.1 & -0.1
\end{array}\right], \\
N_{2} & =\left[\begin{array}{cc}
0.1 & 0.1 \\
-0.1 & 0.1
\end{array}\right], C=\left[\begin{array}{ll}
1 & 0
\end{array}\right] .
\end{aligned}
$$

Figure 1 shows how the shape of the ellipse $\mathcal{E}(Q):=\{x \in$ $\left.\mathbb{R}^{2} \mid x^{\prime} Q^{-1} x \leq 1\right\}$ varies with a rescaling of time $\tau=\frac{1}{\alpha} t$. As explained in [14], PCA for dynamical systems involves projecting onto the subspace spanned by the first $q$ singular vectors of the Gramian. The numerical example shows these can be adjusted almost arbitrarily simply by changing the time unit. This motivates the need for a new Gramian definition. Similarly, for $\alpha>50$, no solution to (4) exists and hence no methods revolving around the bilinear system Gramians could be used.

\section{B. Definition and computation of new Gramians}

The approach we take in this work is to exploit the presence of input constraints to define energy results in terms of simple LMIs.

Lemma 1: Consider a realization $\left(A, N_{1}, \ldots, N_{m}, C, R\right)$ and constant matrices $P, Q \succ 0$, then

$$
\begin{array}{r}
\left(A+\sum_{i=1}^{m} u_{i}(t) N_{i}\right) P+P\left(A+\sum_{i=1}^{m} u_{i}(t) N_{i}\right)^{\prime} \\
+R R^{\prime} \prec 0, \\
\left(A+\sum_{i=1}^{m} u_{i}(t) N_{i}\right)^{\prime} Q+Q\left(A+\sum_{i=1}^{m} u_{i}(t) N_{i}\right) \\
+C^{\prime} C \prec 0
\end{array}
$$

hold for all $u \in \mathcal{U}^{m}$ if and only if

$$
\begin{aligned}
& \tilde{A}_{i} P+P \tilde{A}_{i}^{\prime}+R R^{\prime} \prec 0, \\
& \tilde{A}_{i}^{\prime} Q+Q \tilde{A}_{i}+C^{\prime} C \prec 0,
\end{aligned}
$$

for all $i \in\left\{1, \ldots, 2^{m}\right\}$, where $\tilde{A}_{i}$ is the $i^{\text {th }}$ component in the $2^{m}$-tuple $\mathcal{A}:=\left(A \pm N_{1} \pm \ldots \pm N_{m}\right)$. Furthermore, this implies that

1) if $w=0$, then the energy in the output $y$ for initial condition $x_{0}$ is bounded from above according to

$$
\max _{u \in \mathcal{U}^{m}}\|y\|_{2}^{2}<x_{0}^{\prime} Q x_{0}, \quad \forall x_{0} \in \mathbb{R}^{n} ;
$$

2) the minimum energy of the disturbance $w$ for all input sequences $u \in \mathcal{U}^{m}$ required to drive the system from $x(-\infty)=0$ to $x(0)=x_{0}$ is bounded from below according to

$\forall u \in \mathcal{U}^{m}, \forall x_{0} \in \mathbb{R}^{n}: \min _{w \in \mathcal{L}_{2}[-\infty, 0)}\|w\|_{2}^{2}>x_{0}^{\prime} P^{-1} x_{0}$,

where $\mathcal{L}_{2}(-\infty, 0]$ is the space of Lebesgue measurable functions defined on the interval $(-\infty, 0]$.

Proof: See Appendix.

Note that if any feasible input sequence results in an unbounded energy, neither Gramian will exist. Quadratic stability has been widely used to assess the stability and performance of uncertain linear systems [18] because it facilitates the use of LMIs for the construction of Lyapunov functions. We are therefore constrained to considering only systems that are input independent globally quadratically stable.

Definition 1: A realization is input independent globally quadratically stable (IIGQS) if the origin is a globally quadratically stable equilibrium point [18, p. 61] for all admissible input sequences if $w=0$. 
As an example of when this is the case, think back to the paint mixing example. Red and white paint will mix to become pink, i.e. reach equilibrium, independent of how the mixture is stirred. Of course, the rate at which the equilibrium is reached will change.

Definition 2: A matrix $P \succ 0, P \in \mathbb{R}^{n \times n}$ satisfying (6a) is referred to as a D-Gramian of the realization $\left(A, N_{1}, \ldots, N_{m}, C, R\right)$.

Definition 3: A matrix $Q \succ 0, Q \in \mathbb{R}^{n \times n}$ satisfying (6b) is referred to as an E-Gramian of the realization $\left(A, N_{1}, \ldots, N_{m}, C, R\right)$.

Notice that if a D-Gramian $P$ and an E-Gramian $Q$ exist for a given realization, then $\alpha P, \alpha Q$ will be $\mathrm{D}$ Gramians and E-Gramians, respectively, for the system under a time transformation $\tau=\frac{1}{\alpha} t$. This implies that a simple time transformation cannot cause a reorientation of either Gramian. This is a significant advantage over the bilinear Gramians discussed in Section III-A. As a final remark, we reiterate the point that existence of a D-Gramian or an EGramian neither implies nor is implied by existence of a solution to (3).

\section{Model Reduction}

For a given realization, the feasible set of positive definite solutions to (6) describe the set of $P, Q$ satisfying energy bounds (7). It follows that D-Gramians and E-Gramians for a given realization are not unique. In this section we assume that we are given such quantities. In the seminal work by Moore [14], a Gramian-based model reduction approach was proposed. Here we apply these results to the given DGramian and E-Gramian and exploit results from [19] on balanced truncation for linear parameter-varying systems to define a model reduction algorithm for constrained-input homogeneous-in-the-state bilinear systems. In Section III-D we discuss the appropriate selection of a D-Gramian and EGramian.

Proposition 1: Given a realization $\left(A, N_{1}, \ldots, N_{m}, C, R\right)$, a D-Gramian $P$, an E-Gramian $Q$ and a state transformation matrix $U \in \mathbb{R}^{n \times n}$, then $\left(U A U^{-1}, U N_{1} U^{-1}, \ldots, U N_{m} U^{-1}, C U^{-1}, U R\right) \quad$ is the realization of the transformed system and

$$
\tilde{P}:=U P U^{\prime} \quad \text { and } \quad \tilde{Q}:=\left(U^{\prime}\right)^{-1} Q U^{-1}
$$

are D-Gramians and E-Gramians of the transformed system, respectively.

Proof: See Appendix.

Definition 4: A realization is an internally balanced disturbed realization with respect to a D-Gramian $P$ and an E-Gramian $Q$ if and only if $P$ and $Q$ are equal, diagonal and positive definite.

Note that existence of the D-Gramian and E-Gramian implies that two positive definite matrices exist satisfying (6a) and (6b), respectively. In this case, there always exists a balancing transformation $U$ such that $U P U^{\prime}=\left(U^{\prime}\right)^{-1} Q U^{-1}$, which follows from [20, p. 75]. This implies that there always exists a state transformation such that the transformed realization is an internally balanced one. The extension to the case where some modes contribute nothing to the output or cannot be reached by a feasible disturbance sequence is not considered. To compute a transformation matrix for the positive definite case, the following process can be used [20, p. 78]:

Algorithm 1: Given a D-Gramian $P$ and an E-Gramian $Q$ :

1) Find $S \in \mathbb{R}^{n \times n}$ such that $P=S^{\prime} S$;

2) Perform the decomposition $S Q S^{\prime}=V \Sigma^{2} V^{\prime}$;

3) Set $U^{-1}=S^{\prime} V \Sigma^{-1 / 2}$, then the transformed realization, which from Proposition 2 can be written $\left(U A U^{-1}, U N_{1} U^{-1}, \ldots, U N_{m} U^{-1}, C U^{-1}, U R\right)$, is internally balanced with respect to the D-Gramian $\tilde{P}$ and E-Gramian $\tilde{Q}$, where $\tilde{P}=\tilde{Q}=\Sigma$.

Theorem 1: Consider an internally balanced disturbed realization $\left(A, N_{1}, \ldots, N_{m}, C, R\right)$ with respect to a DGramian $P$ and an E-Gramian $Q$, partitioned as follows:

$$
\begin{array}{r}
A=\left[\begin{array}{ll}
A_{11} & A_{12} \\
A_{21} & A_{22}
\end{array}\right], \quad N_{i}=\left[\begin{array}{cc}
N_{11}^{i} & N_{12}^{i} \\
N_{21}^{i} & N_{22}^{i}
\end{array}\right], \\
C=\left[\begin{array}{ll}
C_{1} & C_{2}
\end{array}\right], \quad R=\left[\begin{array}{l}
R_{1} \\
R_{2}
\end{array}\right], \quad P=Q=\Sigma=\left[\begin{array}{cc}
\Sigma_{1} & 0 \\
0 & \Sigma_{2}
\end{array}\right],
\end{array}
$$

where $A_{11}, N_{11}^{i} \in \mathbb{R}^{q \times q}, A_{12}, N_{12}^{i} \in \mathbb{R}^{q \times(n-q)}, A_{21}, N_{21}^{i} \in$ $\mathbb{R}^{(n-q) \times q}, A_{22}, N_{22}^{i} \in \mathbb{R}^{(n-q) \times(n-q)}, C_{1} \in \mathbb{R}^{p \times q}, C_{2} \in$ $\mathbb{R}^{p \times(n-q)}, R_{1} \in \mathbb{R}^{q \times r}, R_{2} \in \mathbb{R}^{(n-q) \times r}$ and $\Sigma_{1}:=$ $\operatorname{diag}\left(\sigma_{1}, \cdots, \sigma_{q}\right), \Sigma_{2}:=\operatorname{diag}\left(\sigma_{q+1}, \cdots, \sigma_{n}\right), \Sigma_{1}, \Sigma_{2} \succ$ 0 . If the undisturbed realization $\left(A, N_{1}, \cdots, N_{m}, C, R\right)$ is IIGQS, then

1) the truncated disturbed realization $\left(A_{11}, N_{11}^{1}, \cdots, N_{11}^{m}, C_{1}, R_{1}\right)$ is IIGQS;

2) the truncated disturbed realization $\left(A_{11}, N_{11}^{1}, \cdots, N_{11}^{m}, C_{1}, R_{1}\right)$ is internally balanced with respect to the D-Gramian $\hat{P}$ and E-Gramian $\hat{Q}$, where $\hat{P}=\hat{Q}=\Sigma_{1}$;

3) if $\sigma_{1} \geq \sigma_{2} \geq \cdots \geq \sigma_{q}>\sigma_{q+1} \geq \cdots \geq \sigma_{n}>0$, then:

$$
\begin{array}{r}
\max _{u \in \mathcal{U}^{m}} \max _{\substack{w \in \mathcal{L}_{2}[0, \infty) \\
w \neq 0}} \frac{\int_{0}^{\infty}(y(t)-\hat{y}(t))^{\prime}(y(t)-\hat{y}(t)) \mathrm{d} t}{\int_{0}^{\infty} w^{\prime}(t) w(t) \mathrm{d} t} \\
\leq 2 \sum_{j=q+1}^{n} \sigma_{j} ;
\end{array}
$$

Proof: The realization $\left(A, N_{1}, \ldots, N_{m}, C, R\right)$ is IIGQS and internally balanced, so by definition the E-Gramian is diagonal, positive semi-definite and must satisfy

$$
\begin{aligned}
{\left[\begin{array}{cc}
\tilde{A}_{11}^{i} & \tilde{A}_{12}^{i} \\
\tilde{A}_{21}^{i} & \tilde{A}_{22}^{i}
\end{array}\right]^{\prime}\left[\begin{array}{cc}
\Sigma_{1} & 0 \\
0 & \Sigma_{2}
\end{array}\right]+} & {\left[\begin{array}{cc}
\Sigma_{1} & 0 \\
0 & \Sigma_{2}
\end{array}\right]\left[\begin{array}{cc}
\tilde{A}_{11}^{i} & \tilde{A}_{12}^{i} \\
\tilde{A}_{21}^{i} & \tilde{A}_{22}^{i}
\end{array}\right] } \\
& +\left[\begin{array}{c}
C_{1}^{\prime} \\
C_{2}^{\prime}
\end{array}\right]\left[\begin{array}{ll}
C_{1} & C_{2}
\end{array}\right] \prec 0,
\end{aligned}
$$

for all $i \in\left\{1, \ldots, 2^{m}\right\}$, where

$$
\tilde{A}_{i}:=\left[\begin{array}{cc}
\tilde{A}_{11}^{i} & \tilde{A}_{12}^{i} \\
\tilde{A}_{21}^{i} & \tilde{A}_{22}^{i}
\end{array}\right],
$$

$\tilde{A}_{11}^{i} \in \mathbb{R}^{q \times q}, \tilde{A}_{12}^{i} \in \mathbb{R}^{q \times(n-q)}, \tilde{A}_{21}^{i} \in \mathbb{R}^{(n-q) \times q}, \tilde{A}_{22}^{i} \in$ $\mathbb{R}^{q \times q}, \tilde{A}_{i}$ is the suitably partitioned $i^{\text {th }}$ component in the $2^{m}$ tuple $\mathcal{A}:=\left(A \pm N_{1} \pm \ldots \pm N_{m}\right)$ and $\tilde{A}_{11}^{i}$ is the $i^{\text {th }}$ component 
in the $2^{m}$-tuple $\tilde{\mathcal{A}}:=\left(A_{11} \pm N_{11}^{1} \pm \ldots \pm N_{11}^{m}\right)$. Sylvester's criterion [21] implies that

$$
\tilde{A}_{11}^{i^{\prime}} \Sigma_{1}+\Sigma_{1} \tilde{A}_{11}^{i}+C_{1}^{\prime} C_{1} \prec 0,
$$

for all $i \in\left\{1, \ldots, 2^{m}\right\}$. Hence $\Sigma_{1}$ is an E-Gramian for the reduced realization. By definition, $\Sigma_{1} \succ$ 0 , so Corollary 1 implies that the truncated realization $\left(A_{11}, N_{11}^{1}, \ldots, N_{11}^{m}, C_{1}, R_{1}\right)$ is IIGQS, thus confirming part 1 . Likewise the D-Gramian by definition must satisfy

$$
\begin{array}{r}
{\left[\begin{array}{cc}
\tilde{A}_{11}^{i} & \tilde{A}_{12}^{i} \\
\tilde{A}_{21}^{i} & \tilde{A}_{22}^{i}
\end{array}\right]\left[\begin{array}{cc}
\Sigma_{1} & 0 \\
0 & \Sigma_{2}
\end{array}\right]+} \\
+\left[\begin{array}{cc}
\Sigma_{1} & 0 \\
0 & \Sigma_{2}
\end{array}\right]\left[\begin{array}{cc}
\tilde{A}_{11}^{i} & \tilde{A}_{12}^{i} \\
\tilde{A}_{21}^{i} & \tilde{A}_{22}^{i}
\end{array}\right]^{\prime} \\
+\left[\begin{array}{c}
R_{1} \\
R_{2}
\end{array}\right]\left[\begin{array}{ll}
R_{1}^{\prime} & R_{2}^{\prime}
\end{array}\right] \prec 0,
\end{array}
$$

which, again by Sylvester's criterion [21], implies that

$$
\tilde{A}_{11}^{i} \Sigma_{1}+\Sigma_{1} \tilde{A}_{11}^{i^{\prime}}+R_{1} R_{1}^{\prime} \prec 0,
$$

for all $i \in\left\{1, \ldots, 2^{m}\right\}$. Hence $\Sigma_{1}$ is also a D-Gramian for the reduced realization. In conjunction with the fact that $\Sigma_{1}$ is diagonal, this shows that the reduced realization is internally balanced with respect to the D-Gramian and EGramian $\hat{P}, \hat{Q}=\Sigma_{1}$, thus confirming part 2 .

Due to the fact that a positive-definite D-Gramian and EGramian exist, Lemma 1 implies that (5) holds for all $u \in$ $\mathcal{U}^{m}$. In conjunction with the results of [16, Chap. 4] and [19, Lem. 4.1], this implies part 3.

Note the condition that two identical singular values must not be separated in the reduction procedure. For an explanation of the necessity of such a condition, see [16, p. 159].

To summarize, the model reduction scheme, given a D-Gramian $P$ and an E-Gramian $Q$, can be written as a state transformation followed by a truncation. The state transformation yields an internally balanced disturbed realization with respect to the transformed Gramians, while the truncation then throws away the dynamics that are not very important in the disturbance-error $\mathcal{L}_{2^{-}}$ gain sense. If $U \in \mathbb{R}^{n \times n}$ is the balancing transformation, $I \in \mathbb{R}_{q \times q}$ is the identity matrix and 0 is a matrix of zeros of compatible dimension, then the reducedorder realization is $\left(\left[\begin{array}{ll}I & 0\end{array}\right] U A U^{-1}\left[\begin{array}{ll}I & 0\end{array}\right]^{\prime},\left[\begin{array}{ll}I & 0\end{array}\right] U N_{1} U^{-1}\left[\begin{array}{ll}I & 0\end{array}\right]^{\prime}\right.$,

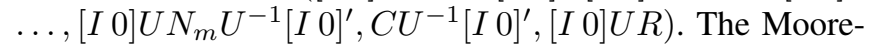
Penrose pseudo-inverse has the following properties [15]:

1) $\left[\begin{array}{ll}I & 0\end{array}\right]^{+}=\left[\begin{array}{ll}I & 0\end{array}\right]^{\prime}$;

2) If $A$ is full rank, $B$ is surjective and $A$ and $B$ are of compatible dimensions, then $(B A)^{+}=A^{-1} B^{+}$.

These properties imply that if $T:=\left[\begin{array}{ll}I & 0\end{array}\right] U$ then $T^{+}=U^{-1}\left[\begin{array}{ll}I & 0\end{array}\right]^{\prime}$. This allows us to write the realization of the reduced system in the more compact form $\left(T A T^{+}, T N_{1} T^{+}, \ldots, T N_{m} T^{+}, C T^{+}, T R\right)$.

\section{Choice of D-Gramian and E-Gramian}

Thus far we have assumed existence of a D-Gramian and an E-Gramian. As commented in Section III-C these are not unique. In this section we discuss suitable choices for such Gramians. From Theorem 1 we know that the upper bound of the error is related to the final $n-q$ singular values of the balanced Gramian, therefore the aim should be to find a D-Gramian $P$ and an E-Gramian $Q$ such that these $n-q$ singular values are minimized. The singular values of the transformed Gramians are the square root of the singular values of $P Q[20$, p. 75] ordered from largest to smallest, so the problem becomes

$$
\min _{P, Q \succ 0} \operatorname{trace}(P Q) \quad \text { subject to (6). }
$$

Although the LMI constraints are convex, the cost is not [19]. In [19] the authors propose an algorithm for computing a locally optimal solution to such a problem, based on recursively solving several LMI constrained optimization problems:

Algorithm 2: Given an initial guess $Q_{0}$ for the E-Gramian that satisfies (6b), set $i=0$ then

1) Solve $P_{i}=\arg \min _{P \succ 0} \operatorname{trace}\left(P Q_{i}\right)$ subject to $\tilde{A}_{i} P+$ $P \tilde{A}_{i}^{\prime}+R R^{\prime} \preceq-\delta I$

2) Set $i \leftarrow i+1$ and solve $Q_{i}=\arg \min _{Q \succ 0} \operatorname{trace}\left(P_{i} Q\right)$ subject to $\tilde{A}_{i}^{\prime} Q+Q \tilde{A}_{i}+C^{\prime} C \preceq-\delta I$

3) Repeat steps 1 and 2 until the decrease in cost trace $\left(P_{i} Q_{i+1}\right)$ reduces below a certain tolerance.

The presence of a small $\delta$, typically of order $1 \times 10^{-5}$, in the LMI constraints ensures the LMIs are strictly 'less than' as opposed to 'less than or equal to'. To compute the initial guess $Q_{0}$ we solve $Q_{0}:=\arg \min _{Q \succ 0} \operatorname{trace}(Q)$ subject to (6b). The LMI constrained optimization to be solved to initialize the problem, and the ones to be solved at each iteration, are convex, although the algorithm itself will only compute a locally optimal solution to (8) [19].

The proposed algorithm requires the successive solution of LMI constrained optimization problems. Efficient interior point algorithms exist for the construction of solutions to such problems [18] with the additional structure of the specific problem being examined in [22] and a polynomial time algorithm being proposed in [23]. In contrast, the solution to a generalized Lyapunov equation required for methods revolving around the bilinear system Gramians is known explicitly [13]. However, it does involve $m+2$ Kronecker products between matrices of size $n \times n$ and hence for largescale problems storage requirements are large. It is worth noting that model reduction algorithms are implemented offline and hence the computational requirements are not of fundamental importance but are still worthy of comment.

\section{CASe STudy: Solar COllector Plant}

In the past 30 years there has been considerable interest in the use of solar power as a viable alternative to nonrenewable sources such as coal and natural gas. One form of solar collector uses a mirrored parabolic trough to focus sunlight onto a receiver pipe carrying a heat transfer fluid. The aim is to heat this fluid to a given target outlet temperature. It is then passed through a heat exchanger in order to extract the energy. The target outlet temperature is the optimum safe operating temperature of this heat exchanger. 
In order to be an implementable solution, the solar collector must be capable of regulating the temperature at the outlet of the receiver pipe despite fluctuations in the sunlight. The control variable is the flow rate. The solar collector plant from [5] is a continuous-time, homogeneous-in-the-state, bilinear system with the incident radiation taking the form of an additive disturbance and hence has a disturbed realization $\left(A, N_{1}, C, R\right)$ with state dimension $n=41$ where:

$$
\begin{aligned}
& A=\left[\begin{array}{ccc}
A_{1} & 0_{20 \times 1} & 0_{20 \times 20} \\
0_{1 \times 20} & A_{2} & 0_{1 \times 20} \\
0_{20 \times 20} & 0_{20 \times 1} & 0_{20 \times 20}
\end{array}\right], \\
& N_{1}=\operatorname{diag}\left(\left[\begin{array}{lll}
-\alpha 1_{1 \times 20} & 0 & -\alpha 1_{1 \times 20}
\end{array}\right]\right)
\end{aligned}
$$

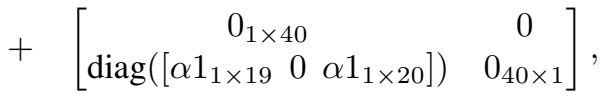

$$
\begin{aligned}
& C=\left[\begin{array}{lll}
0_{1 \times 19} & 1 & 0_{1 \times 21}
\end{array}\right], \quad R=\left[\begin{array}{cc}
R_{11} & R_{12} \\
R_{21} & 0 \\
0_{20 \times 1} & 0_{20 \times 1}
\end{array}\right],
\end{aligned}
$$

and $A_{1}=-\beta_{1} I_{20 \times 20}, A_{2}=-1-\beta_{2}, R_{11}=\beta_{1} T_{1} 1_{20 \times 1}$, $R_{21}=\beta_{2} T_{2}, R_{12}=\gamma_{1} 1_{20 \times 1}, \alpha=8.22 \times 10^{-3} \mathrm{~kg}^{-1}$, $\beta_{1}=1.19 \times 10^{-3} \mathrm{~s}^{-1}, \beta_{2}=5 \mathrm{~s}^{-1}, \gamma=0.541 \mathrm{Ks}^{-1}, T_{1}=$ $303.15 \mathrm{~K}$ and $T_{2}=375.15 \mathrm{~K}$. This model stems from the discretization of the partial differential equations governing the heat transfer of a fluid in a pipe with the coarseness of discretization chosen as a trade-off between model size and accuracy [5]. The input constraint set $\mathcal{U}=[0.8,8]$. This can be transformed to the standard form with the substitution $u=4.4+3.6 \tilde{u}$. The realization then becomes $\left(A+4.4 N_{1}, 3.6 N_{1}, C, R\right)$.

In order to highlight the problems associated with methods revolving around the bilinear system Gramians detailed in Section IIIA, we consider the use of the model reduction scheme from [7] applied to models with range of time transformations $\tau=\frac{1}{\alpha} t$. Figure 2 shows the results. The reduced-order models with $q=10$ are simulated for a given input and disturbance trajectory and the results compared to the original plant. As $\alpha$ is increased, the similarity between the reduced order model and the plant decreases. For $\alpha=$ 60 , no positive definite solution exists to the generalized Lyapunov equation (4) and as such the scheme cannot be implemented. We stress that this means that had the modeling been done in minutes instead of seconds, approaches using the bilinear system Gramians from [12] cannot be applied.

Now we consider the performance of the new method introduced in Section IIIC. An E-Gramian $Q$ and a DGramian $P$ are constructed using Algorithm 2 with $\delta=$ $1 \times 10^{-5}$ and it requires 4 iterations before the cost of successive iterations are the same to a relative tolerance of $1 \times 10^{-6}$. The singular values of the balanced Gramian are plotted in Figure 3. It is clear that the first few modes contain most of the system energy for all input sequences. Figure 4 shows the performance of a range of models with different numbers of states to a range of different input sequences. The initial conditions used for the different plots are simply $x_{0}=$ $465 \times 1_{41 \times 1}$, where $1_{41 \times 1}$ is a vector of ones representing

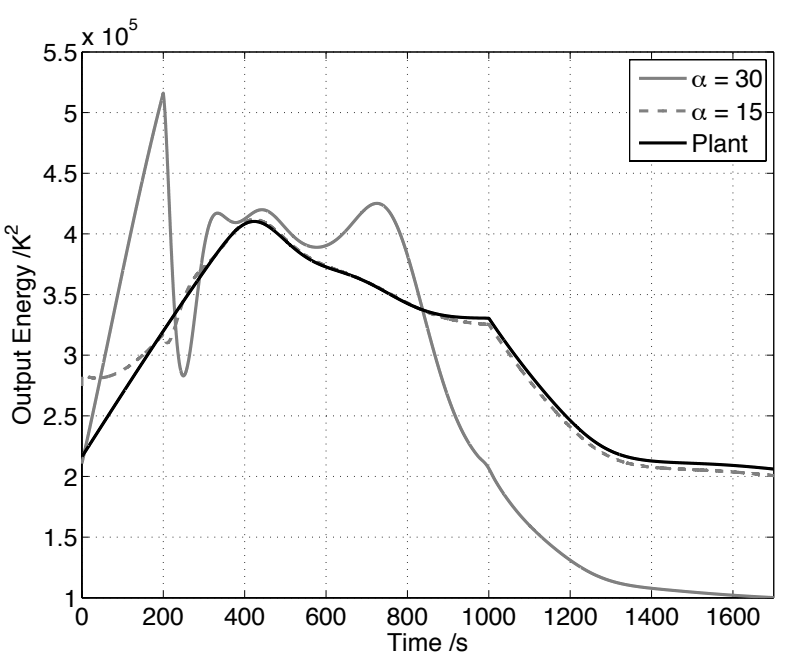

Fig. 2. Comparison of three reduced order models with $q=10$ generated using the balanced truncation method from [7] with $u(t)=0.8$ for all $t \in[0,200), u(t)=8$ for all $t \in[200,1750), w_{2}(t)=1.4$ for all $t \in[0,1000)$ and $w_{2}(t)=0.6$ for all $t \in[1000,1750)$ for two different time transformations $\tau=\frac{1}{\alpha} t$.

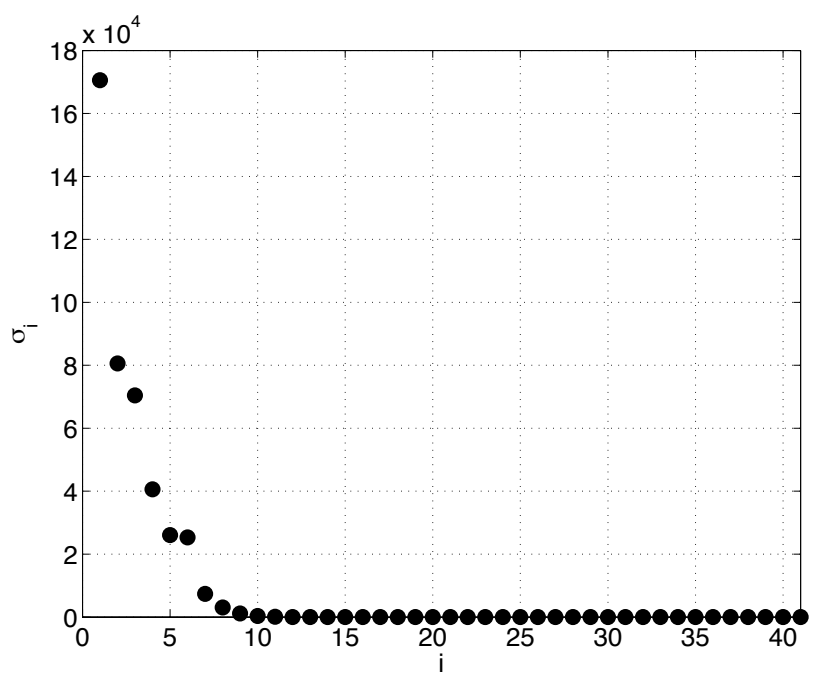

Fig. 3. Figure showing the 41 singular values $\sigma_{i}$ of $P Q$

the case were the temperature is $465 \mathrm{~K}$ throughout the fluid. The output is the outlet temperature, measured in Kelvin and hence the output energy is of order $10^{5}$. For the reducedorder models, $\hat{x}(0)=T x_{0}$. The disturbance is of the form $w=\left[\begin{array}{ll}1 & w_{2}\end{array}\right]^{\prime}$ where $w_{2}$ is a scalar function. This is because the first disturbance represents a change in ambient temperatures, which is a very small effect, whereas the second is the incident radiation. This is a much larger effect and obviously one that is critical to the process. Clearly the reduced-order models perform very well and suggest that they could be used for simulation or control design. Although for this example the original state dimension seems small, a reduction from 41 states to 7 can significantly decrease the computation time and hence the lowest achievable sampling time for a receding 


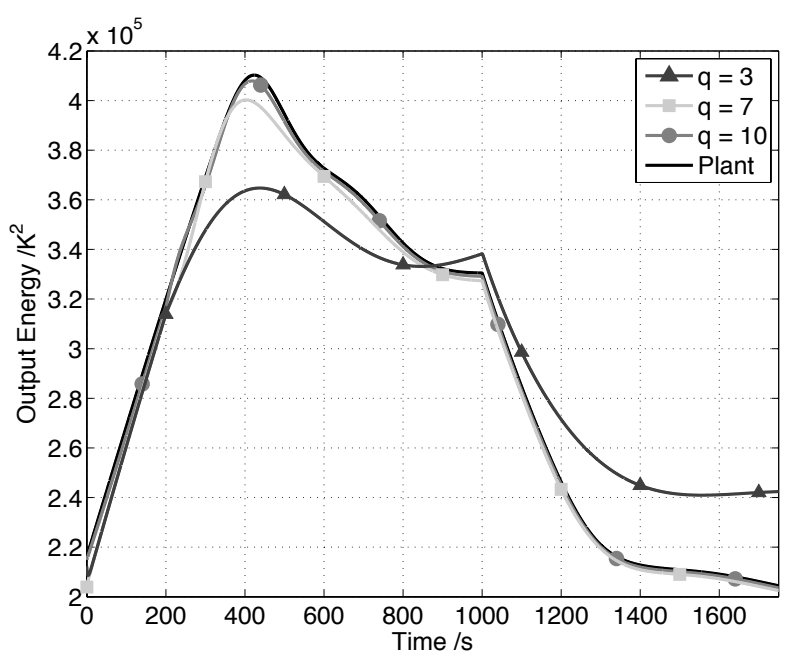

(a) $u(t)=0.8$ for all $t \in[0,200), u(t)=8$ for all $t \in[200,1750)$, $w_{2}(t)=1.4$ for all $t \in[0,1000)$ and $w_{2}(t)=0.6$ for all $t \in$ $[1000,1750)$.

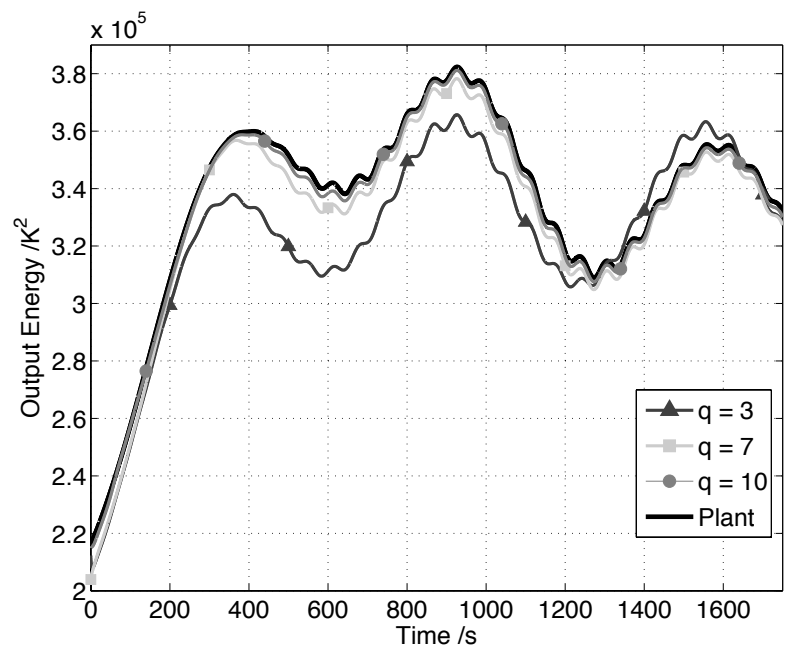

(b) $u(t)=4.4+3.6 \cos (0.1 t)$ and $w_{2}(t)=1+0.4 \sin (0.01 t)$.

Fig. 4. Comparison of plant to three reduced order models computed with the new method for a range of disturbances and inputs.

horizon controller.

Figure 5 shows the performance of the balanced truncation approach from [7] based upon the bilinear system Gramians discussed in Section III-A. Interestingly for the $q=10$ case, the model is almost identical to the plant, but unlike the new approach $q=7$ does not achieve even remotely similar results.

All computations were performed on a $2.66 \mathrm{GHz}$ Quad Core Intel Xeon CPU machine, IEEE double precision arithmetic with relative machine precision $\epsilon=2.22 \times 10^{-16}$. The YALMIP interface [24] to MATLAB 7.4 with SDPT3 optimization toolbox [25] was used. The proposed reduction algorithm took approximately 1 minute to complete.

\section{CONCLUSions}

This paper describes an approach for the model reduction of IIGQS homogeneous-in-the-state bilinear systems. This

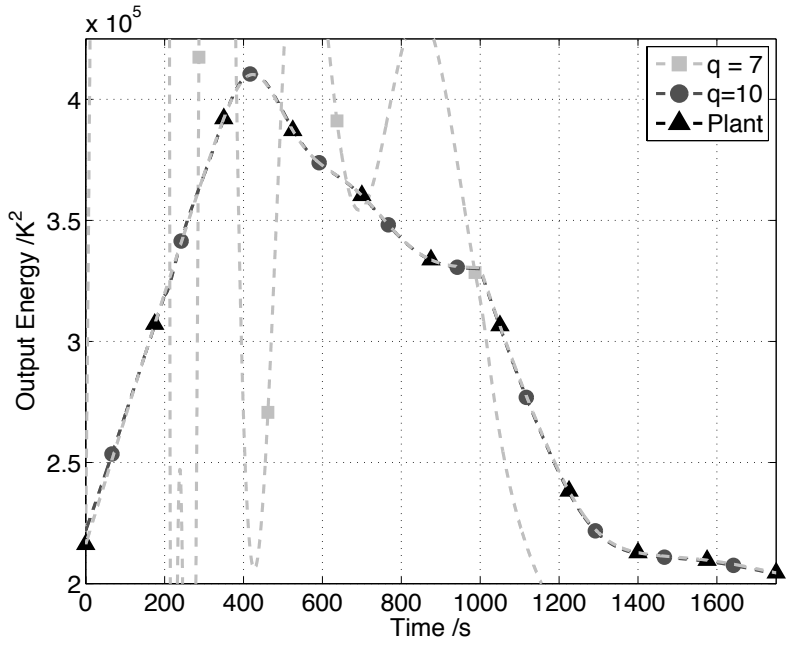

(a) $u(t)=0.8$ for all $t \in[0,200), u(t)=8$ for all $t \in[200,1750)$, $w_{2}(t)=1.4$ for all $t \in[0,1000)$ and $w_{2}(t)=0.6$ for all $t \in$ $[1000,1750)$.

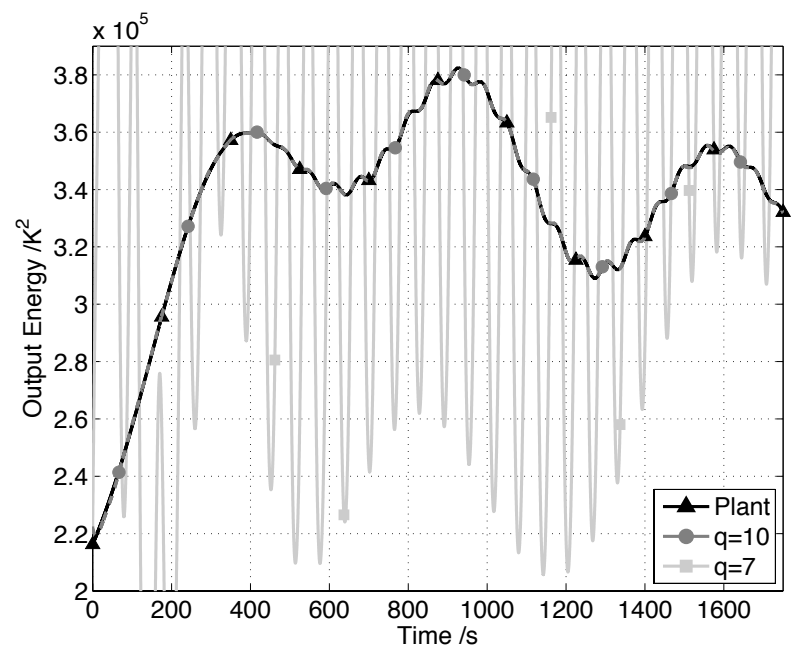

(b) $u(t)=4.4+3.6 \cos (0.1 t)$ and $w_{2}(t)=1+0.4 \sin (0.01 t)$.

Fig. 5. Comparison of plant to three reduced order models computed with the balanced truncation method from [7] for a range of disturbances and inputs.

is an improvement on existing techniques based around the bilinear observability and controllability Gramians, where a simple rescaling in time results in a change in their alignment and so changes the reduction. To counter this, we exploit the input constraints to write energy bounds in terms of linear matrix inequalities. This allows us to define two new Gramians about which a familiar reduction scheme can be based. The new method is shown to outperform an existing method for similar sizes of reduced order model on a numerical example.

A drawback of this approach is that the complexity of the problem increases exponentially with the number of inputs. This can prohibitively increase the problem size. For systems with a large number of states, single Lyapunov equations can be solved efficiently with Krylov subspace methods [26]. Similar algorithms will need to be created for multiple or 
structured Lyapunov equations as we have here. The final aim for future work could be to extend the ideas developed here for homogeneous-in-the-state bilinear systems to more general bilinear systems.

In Section IV it has been shown that a reduced-order model of a solar collector plant with similar disturbance to output properties exists. Control problems involving such a plant are nonlinear and constrained and so receding horizon control has often been applied. A further target for future work would be the design of an efficient receding horizon controller for this real world plant.

\section{APPENDIX}

Proof of Lemma 1 Since $u_{i}(t) \in[-1,1]$ for all $t$,

$$
\begin{array}{r}
\sum_{i=1}^{m} u_{i}(t) N_{i} \in\left\{N \in \mathbb{R}^{n \times n} \mid N=\sum_{i=1}^{m} \alpha_{i} N_{i}, \alpha_{i} \in[-1,1]\right\} \\
\in\left\{N \in \mathbb{R}^{n \times n} \mid N=\sum_{i=1}^{2^{m}} \theta_{i} \tilde{N}_{i}, \theta_{i} \in[0,1], \sum_{i=1}^{2^{m}} \theta_{i}=1\right\} \\
\in \operatorname{Co}\left\{\tilde{N}_{1}, \ldots, \tilde{N}_{m}\right\},
\end{array}
$$

for all $t$, where $\tilde{N}_{i}$ is the $i^{\text {th }}$ component of the $2^{m}$ tuple $\left( \pm N_{1} \pm N_{2} \pm \ldots \pm N_{m}\right)$. For proof of this result, see [27, p. 316]. In conjunction with [28, p. 14], this implies

$$
A+\sum_{i=1}^{m} u_{i}(t) N_{i} \in \operatorname{Co}\left\{\tilde{A}_{1}, \ldots, \tilde{A}_{2^{m}}\right\}=: \mathcal{B} .
$$

In order to satisfy (5a) and (5b) it is necessary and sufficient to find a $P$ and $Q$ satisfying (6a) and (6b) [18, pp. 79,86]. It follows that the set of feasible trajectories with initial condition $x_{0}=x(0)$ can be written as a polytopic linear differential inclusion (PLDI)

$$
\dot{x}(t) \in \mathcal{B} x(t)+R w(t), \quad y(t)=C x(t) .
$$

The energy results (7a) and (7b) are then equivalent to those given for PLDIs in [18, pp. 79,86].

Proof of Proposition 1 By writing $\tilde{x}(t)=U x(t)$, the transformed realization is clear. Consider the set of LMIs that the E-Gramian $\tilde{Q}$ of the transformed realization must satisfy:

$U \tilde{A}_{i}^{\prime} U^{\prime} \tilde{Q}+\tilde{Q} U \tilde{A}_{i} U^{\prime}+U C^{\prime} C U^{\prime} \prec 0, \quad \forall i \in\left\{1, \ldots, 2^{m}\right\}$.

Substituting $\tilde{Q}=\left(U^{\prime}\right)^{-1} Q U^{-1}$, pre-multiplying by $U^{-1}$ and post-multiplying by $U$ yields the desired result. The proof of the transformed D-Gramian follows in the same fashion.

\section{REFERENCES}

[1] C. Bruni, G. DiPillo, and G. Koch, "Bilinear systems: An appealing class of 'nearly linear' systems in theory and applications," IEEE Transactions on Automatic Control, vol. 19, pp. 334-348, 1974.

[2] R. R. Mohler, Bilinear control processes with application to engineering, ecology and medicine. Academic Press, 1973.

[3] Z. Bai and D. Skoogh, "A projection method for model reduction of bilinear dynamical systems," Linear Algebra and its Applications, vol. 415, pp. 406-425, 2006.

[4] G. Mathew, I. Mezić, S. Grivopoulos, U. Vaidya, and L. Petzold, "Optimal control of mixing in Stokes fluid flows," Journal of Fluid Mechanics, vol. 580, pp. 261-281, 2007.
[5] M. J. Tenny, J. B. Rawlings, and S. J. Wright, "Closed-loop behavior of nonlinear model predictive control," Process Systems Engineering, vol. 50, pp. 2142-2154, 2004.

[6] J. M. A. Scherpen, "Balancing for nonlinear systems," Systems and Control Letters, vol. 21, no. 2, pp. 143-153, 1993.

[7] C. S. Hsu, U. B. Desai, and C. A. Crawley, "Realization algorithms and approximation methods for bilinear systems," in Proceedings of IEEE Conference on Decision and Control, Texas, USA, 1983.

[8] L. Zhang and J. Lam, "On $\mathrm{H}_{2}$ model reduction of bilinear systems," Automatica, vol. 38, pp. 205-216, 2002.

[9] C. Hartmann, A. Zueva, and B. Schafer-Bung., "Balanced model reduction of bilinear systems with applications to positive systems.," Submitted to SIAM Journal of Control and Optimization, 2010.

[10] W. Gray and J. Mesko, "Energy function and algebraic Gramians for bilinear systems.," in Proc. of The 4th IFAC Nonlinear Control Design Symposium, The Netherlands, 1998.

[11] S. Djennoune and M. Bettayeb, "On the structure of energy functions of singularly perturbed bilinear systems," International Journal of Robust and Nonlinear Control, vol. 15, pp. 601-618, 2005.

[12] P. d'Alessandro, A. Isidori, and A. Ruberti, "Realization and structure theory of bilinear dynamical systems," SIAM Journal of Control, vol. 12 , pp. $517-535,1974$.

[13] S. A. Al-Baiyat and M. Bettayeb, "A new model reduction scheme for $k$-power bilinear systems," in Proceedings of IEEE Conference on Decision and Control, Texas, USA, 1993.

[14] B. Moore, "Principal component analysis in linear systems: controllability, observability and model reduction," IEEE Transactions on Automatic Control, vol. 26, pp. 17-32, 1981.

[15] L. Hogben, Handbook of linear algebra. Chapman and Hall, 2007.

[16] G. E. Dullerud and F. Paganini, A course in robust control theory. Springer, 2000.

[17] I. T. Jolliffe, Principal compnent analysis, Second Edition. Springer Series in Statistics, 2002.

[18] S. Boyd, L. E. Ghaoui, E. Feron, and V. Balakrishnan, Linear matrix inequalities in systems and control theory. SIAM Philadelphia, 1994.

[19] G. D. Wood, P. J. Goddard, and K. Glover, "Approximation of linear parameter varying systems," in Proceedings of IEEE Conference on Decision and Control, Kobe, Japan, 1996.

[20] K. Zhou, J. C. Doyle, and K. Glover, Robust and optimal control. Prentice-Hall, 1996.

[21] G. Gilbert, "Positive definite matrices and Sylvester's criterion," The American Mathematical Monthly, vol. 98, pp. 44-46, 1991.

[22] S. Boyd and Q. Yang, "Structured and simultaneous Lyapunov functions for system stability problems," International Journal of Control, vol. 49, no. 6, pp. 2215-2240, 1989.

[23] L. Vandenberghe and S. Boyd, "Polynomial-time algorithm for determining quadratic Lyapunov functions for nonlinear systems," in Proceedings of the European Conference on Circuit Theory and Design, pp. 1065-1068, 1993.

[24] J. Lofberg, "YALMIP: A toolbox for modeling and optimization in MATLAB," in Proceedings of IEEE Symposium on Computer Aided Control System Design, Taipei, Taiwan, 2004.

[25] K. C. Toh, M. J. Todd, and R. H. Tutuncu, "SDPT3: An SC MATLAB software package for semidefinite programming." http://www.math.nus.sg/mattohkc/index.html.

[26] I. M. Jaimoukha and E. M. Kasenally, "Krylov subspace methods for solving large Lyapunov equations," SIAM Journal of Numerical Analysis, vol. 31, pp. 227-251, 1994.

[27] B. Grunbaum, Convex polytopes, Second Edition. Springer, 2002.

[28] A. Brønsted, An introduction to convex polytopes. Springer-Verlag, 1982. 